\title{
INTEGRAL/IBIS nine-year Galactic hard X-ray survey ${ }^{\star \star \star}$
}

\author{
R. Krivonos ${ }^{1,2}$, S. Tsygankov ${ }^{3,4,2}$, A. Lutovinov ${ }^{2}$, M. Revnivtsev ${ }^{2}$, E. Churazov ${ }^{1,2}$, and R. Sunyaev ${ }^{1,2}$ \\ ${ }^{1}$ Max-Planck-Institut für Astrophysik, Karl-Schwarzschild-Str. 1, 85740 Garching bei München, Germany \\ e-mail: krivonos@mpa-garching.mpg.de \\ 2 Space Research Institute, Russian Academy of Sciences, Profsoyuznaya 84/32, 117997 Moscow, Russia \\ 3 Finnish Centre for Astronomy with ESO (FINCA), University of Turku, Väisäläntie 20, 21500 Piikkiö, Finland \\ 4 Astronomy Division, Department of Physics, 90014 University of Oulu, Finland
}

Received 16 May 2012 / Accepted 5 July 2012

\begin{abstract}
Context. The INTEGRAL observatory operating in a hard X-ray/gamma domain has gathered a large observational data set over nine years starting in 2003. Most of the observing time was dedicated to the Galactic source population study, making possible the deepest Galactic survey in hard X-rays ever compiled.

Aims. We aim to perform a Galactic survey that can be used as the basis of Galactic source population studies, and perform mapping of the Milky Way in hard X-rays over the maximum exposure available at $|b|<17.5^{\circ}$.

Methods. We used sky reconstruction algorithms especially developed for the high quality imaging of INTEGRAL/IBIS data.

Results. We present sky images, sensitivity maps, and catalogs of detected sources in the three energy bands 17-60, 17-35, and $35-80 \mathrm{keV}$ in the Galactic plane at $|b|<17.5^{\circ}$. The total number of sources in the reference $17-60 \mathrm{keV}$ band includes 402 objects exceeding a $4.7 \sigma$ detection threshold on the nine-year time-averaged map. Among the identified sources with known and tentatively identified natures, 253 are Galactic objects (108 low-mass X-ray binaries, 82 high-mass X-ray binaries, 36 cataclysmic variables, and 27 are of other types), and 115 are extragalactic objects, including 112 active galactic nuclei and 3 galaxy clusters. The sample of Galactic sources with $S / N>4.7 \sigma$ has an identification completeness of $\sim 92 \%$, which is valuable for population studies. Since the survey is based on the nine-year sky maps, it is optimized for persistent sources and may be biased against finding transients.
\end{abstract}

Key words. surveys - X-rays: general - catalogs

\section{Introduction}

A large fraction of astrophysical phenomena cannot be studied via observations of individual sources, but require instead large statistical studies. The last few decades have provided us with great opportunities for studies of the populations of compact sources (black holes, neutron stars, white dwarfs) in our Galaxy and nearby galaxies.

In particular, surveys of the sky in hard X-rays were performed with the IBIS telescope (Ubertini et al. 2003) of the INTEGRAL observatory (Winkler et al. 2003) and Burst Alert Telescope (BAT; Barthelmy et al. 2005) at the Swift observatory (Gehrels et al. 2004). In contrast to Swift, with a nearly uniform all-sky survey, which is especially useful for studies of active galactic nuclei (AGNs; Tueller et al. 2010; Cusumano et al. 2010; Ajello et al. 2012), the INTEGRAL observatory provides a sky survey with exposures that are deeper in the Galactic plane and has higher angular resolution, which is

\footnotetext{
* Based on observations with INTEGRAL, an ESA project with instruments and science data centre funded by ESA member states (especially the PI countries: Denmark, France, Germany, Italy, Switzerland, Spain), Czech Republic, and Poland, and with the participation of Russia and the USA.

$\star \star$ Table 3 (Catalog) is only available at the CDS via anonymous ftp to cdsarc.u-strasbg. fr (130.79.128.5) or via http://cdsarc.u-strasbg.fr/viz-bin/qcat?J/A+A/545/A27
}

essential in these crowded regions. This makes the Swift/BAT and INTEGRAL/IBIS surveys complementary to each other.

The INTEGRAL observatory has been successfully operating in orbit since its launch in 2002. Over the past few years, INTEGRAL data has allowed us to construct high quality catalogs (Revnivtsev et al. 2004b, 2006; Molkov et al. 2004; Krivonos et al. 2005, 2007b, 2010b; Bird et al. 2006, 2007, 2010), to reveal new types of sources (Courvoisier et al. 2003; Revnivtsev et al. 2003d; Sguera et al. 2006), to calculate the statistics of active galactic nuclei (Sazonov et al. 2007; Bassani et al. 2006; Beckmann et al. 2009), of low mass X-ray binaries (Revnivtsev et al. 2008a), of high mass X-ray binaries (Lutovinov et al. 2005b, 2007; Bodaghee et al. 2007, 2012), and of cataclysmic variables (Revnivtsev et al. 2008b; Scaringi et al. 2010).

In our previous papers (Krivonos et al. 2010a,b), we presented the seven-year hard X-ray all-sky survey in the energy range 17-60 keV based on the improved sky reconstruction method for the IBIS telescope. The sensitivity of the survey was significantly improved by suppressing the systematic noise.

Here we present the selected nine-year averaged sky images, sensitivity maps, and catalog of the sources detected in the Galactic plane $\left(|b|<17.5^{\circ}\right)$ in three energy bands: 17-60, 17-35, and 35-80 keV. This survey is the most sensitive X-ray survey of the Galaxy existing so far and it can be used as: 1) a basis for statistical studies of different types of sources in our 


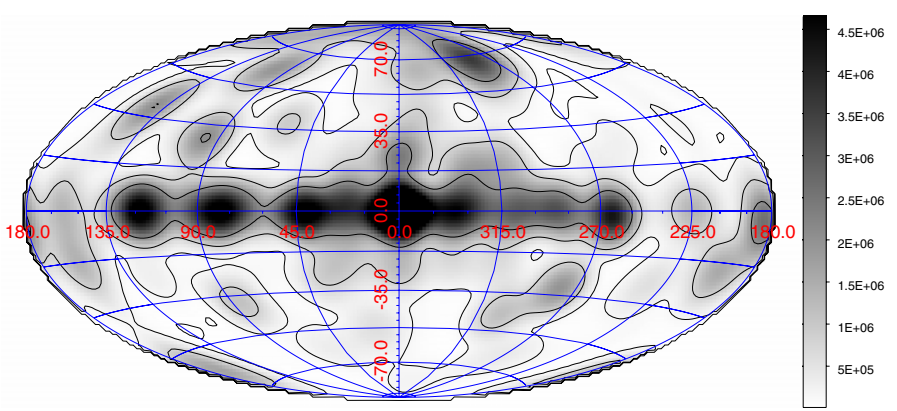

Fig. 1. Dead time-corrected exposure map of the INTEGRAL all-sky survey (January 2011, public data). The grid gap in the Galactic latitude is $17.5^{\circ}$, which is a half-height of the current Galactic survey. Blue contours represent exposure levels of 10, 150, 800, 2000, and $4000 \mathrm{ks}$. The effective exposure in the GC region is $12 \mathrm{Ms}$, which corresponds to $26 \mathrm{Ms}$ of a nominal time.

Galaxy, and 2) a guiding line for new surveys of a new generation of hard X-ray focusing telescopes (e.g. NuSTAR described in Harrison et al. 2010; and Astro-H in Takahashi et al. 2010).

The full set of sky maps is available at the SkyView Virtual Observatory $^{1}$ (McGlynn et al. 1998) and Russian Science Data Center $^{2}$ for the INTEGRAL observatory at the Space Research Institute (IKI), Moscow.

\section{Survey}

To conduct the current Galactic survey, we selected publicly available INTEGRAL data from December 2002 to January 2011 (spacecraft revolutions 26-1013). Every individual INTEGRAL observation with typical exposure time of $2 \mathrm{ks}$ (so called Science Window, $S c W$ ) was analyzed with a specially developed software package (see e.g. Krivonos et al. 2010a, and references therein) to produce sky images in three energy bands: $17-60,17-35$, and $35-80 \mathrm{keV}$. In contrast to our previous surveys, the flux scale in each $S c W$ sky image was adjusted using the flux of the Crab nebula measured in the nearest observations. This procedure was used to account for the ongoing detector degradation and loss of sensitivity at low energies.

In total, we obtained 73489 sky images in each band, which comprises $\sim 132$ Ms of the effective (dead time-corrected) exposure. The survey sky mapping was organized in six overlapping $70^{\circ} \times 35^{\circ}$ Galactic cartesian projections centered on zero Galactic latitude $\left(b=0^{\circ}\right)$ and $l=0^{\circ}, \pm 50^{\circ}, \pm 115^{\circ}$, and $l=180^{\circ}$. The latitude coverage of the survey $|b|<17.5^{\circ}$ was chosen with the IBIS/ISGRI field of view $\left(28^{\circ} \times 28^{\circ}\right)$ and standard $5^{\circ} \times 5^{\circ}$ observational pattern in mind. Thus, we used all observations performed by INTEGRAL in the Galactic plane. Figure 1 illustrates the INTEGRAL exposure map of publicly available observations up to January 2011.

The survey sky coverage versus the $4.7 \sigma$ limiting flux is shown in Fig. 2. The peak sensitivity of the survey is $2.9 \times 10^{-12} \mathrm{erg} \mathrm{s}^{-1} \mathrm{~cm}^{-2}(\sim 0.20 \mathrm{mCrab}$ in $17-60 \mathrm{keV})$ at a $4.7 \sigma$ detection level. The survey covers $90 \%$ of the geometrical area (12680 degrees) down to the flux limit of $2.0 \times 10^{-11} \mathrm{erg} \mathrm{s}^{-1} \mathrm{~cm}^{-2}(\sim 1.41 \mathrm{mCrab})$ and $10 \%$ of the total area down to the flux limit of $4.9 \times 10^{-12} \mathrm{erg} \mathrm{s}^{-1} \mathrm{~cm}^{-2}(\sim 0.30 \mathrm{mCrab})$.

\footnotetext{
1 http://skyview.gsfc.nasa.gov

2 http://hea.iki.rssi.ru/integral
}

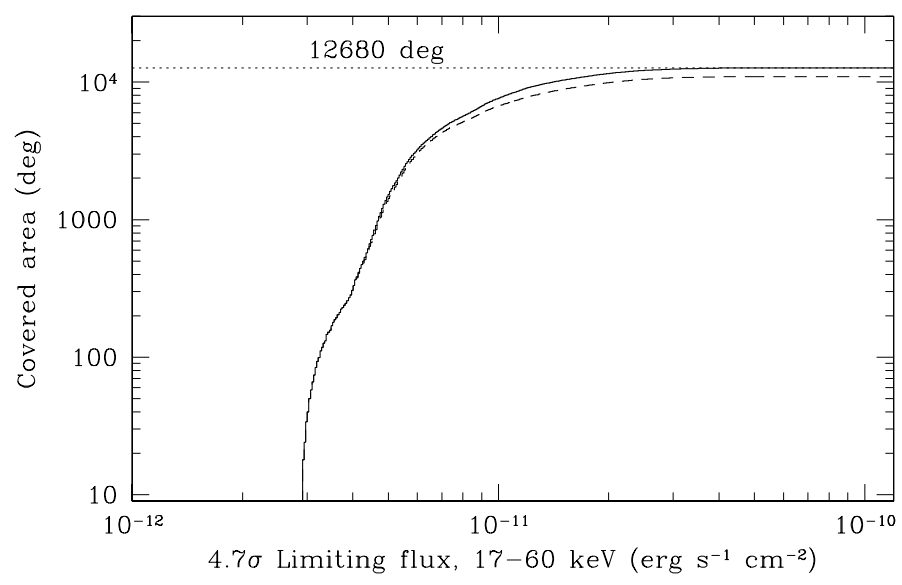

Fig. 2. Sky area as a function of the limiting flux for source detection with a $4.7 \sigma$ significance (solid line). The black dashed curve shows the sky coverage with the masked area around bright sources listed in Table 1 . The dotted line represents the geometrical area.

Table 1. Exclusion radius around bright sources.

\begin{tabular}{lc}
\hline \hline Name & Exclusion radius, deg \\
\hline Crab & 23.2 \\
Sco X-1 & 14.0 \\
Cyg X-1 & 4.8 \\
Cyg X-3 & 3.9 \\
Vela X-1 & 3.3 \\
GX 301-2 & 2.2 \\
GRS 1915+105 & 3.2 \\
\hline
\end{tabular}

\subsection{Systematic noise}

INTEGRAL/IBIS deep sky mosaics are usually affected by a systematic noise caused by the source confusion in the region of GC and by the imperfect sky reconstruction (Krivonos et al. 2010a).

The large field of view (FOV) of the INTEGRAL/IBIS telescope leads to a high probability of having some bright X-ray source within the instrument FOV during any galactic observations. Therefore, to work at the level of Poisson noise, the INTEGRAL/IBIS telescope image reconstruction procedure should have a dynamic range of $10^{3}$ and more, which is very difficult to achieve owing to the imperfect modelling of the mask shadow, the individual pixel sensitivity, the variability of the background pattern, etc.

In spite of the latest version of the IBIS sky image reconstruction allowing us to reach the dynamic range of the images $\sim 10^{3}$, some sky artefacts are still present around bright sources, such as Crab, Sco X-1, Cyg X-1, Cyg X-3, Vela X-1, GX 301-2, and GRS $1915+105$ (see a sky mosaic at $l=+50^{\circ}$ in Fig. 3). To prevent any false detections, we masked out circular regions around bright sources with radii listed in Table 1 . The exclusion radius was chosen to contain all significant $(>4.7 \sigma)$ negative excesses (indicators of the systematic noise, which is assumed to be symmetric) around a given bright source. We note that known sources with $S / N>10 \sigma$ falling inside these regions were included in the source catalog. A rejection of areas with high systematic noise significantly improved the quality of the survey mosaics, which is demonstrated in Fig. 4, where we show a distribution of signal-to-noise $(\mathrm{S} / \mathrm{N})$ values for pixels in the sky mosaic at $l=+50^{\circ}$. As seen from Fig. 4, the masked sky 


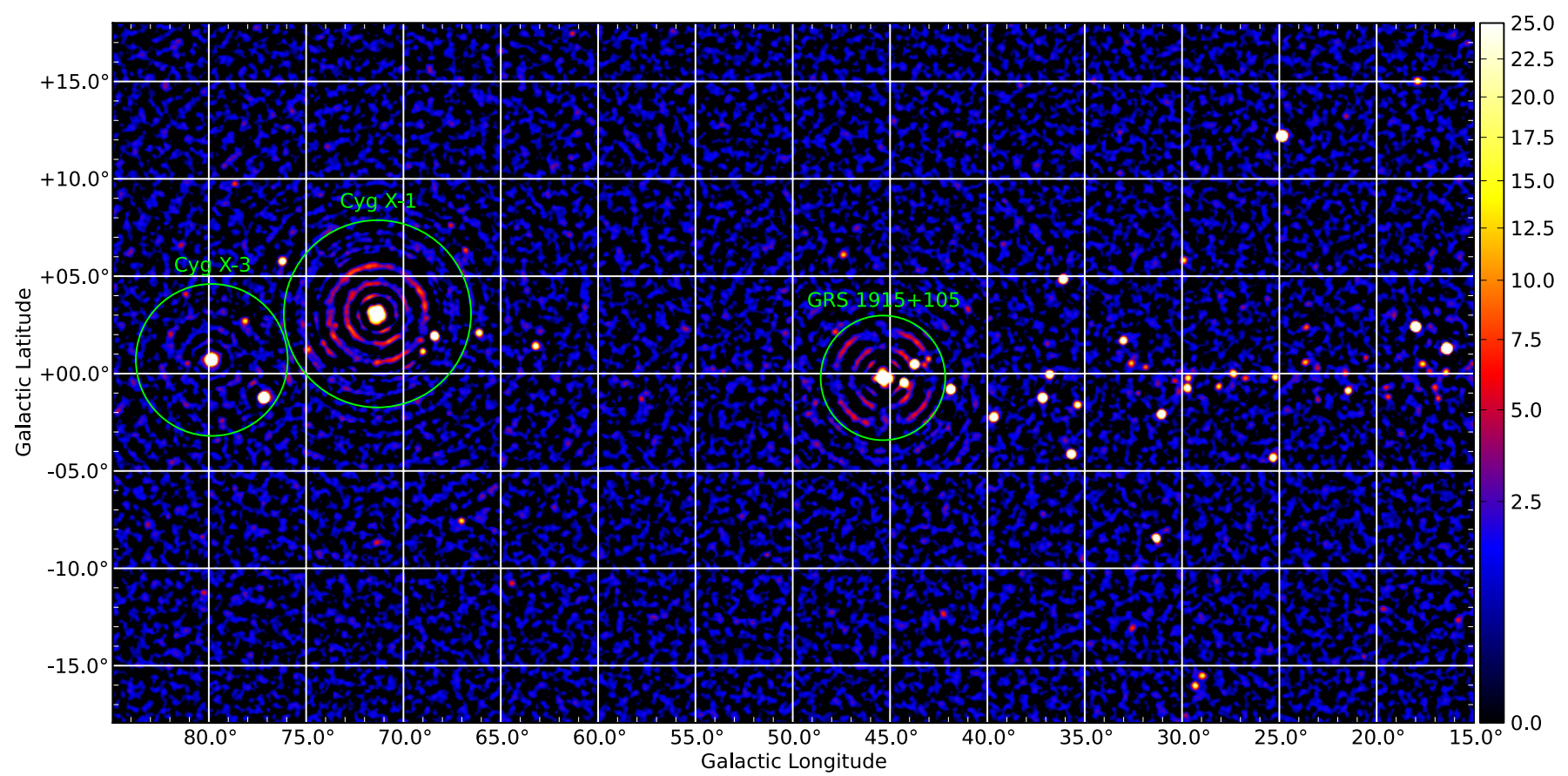

Fig. 3. INTEGRAL/IBIS hard X-ray (17-60 keV) map of the sky region of Cyg X-1, Cyg X-3, and GRS $1915+105$ at $l=+50^{\circ}$ with masked area shown as green circles. The corresponding $\mathrm{S} / \mathrm{N}$ distribution of pixels is shown in Fig. 4.

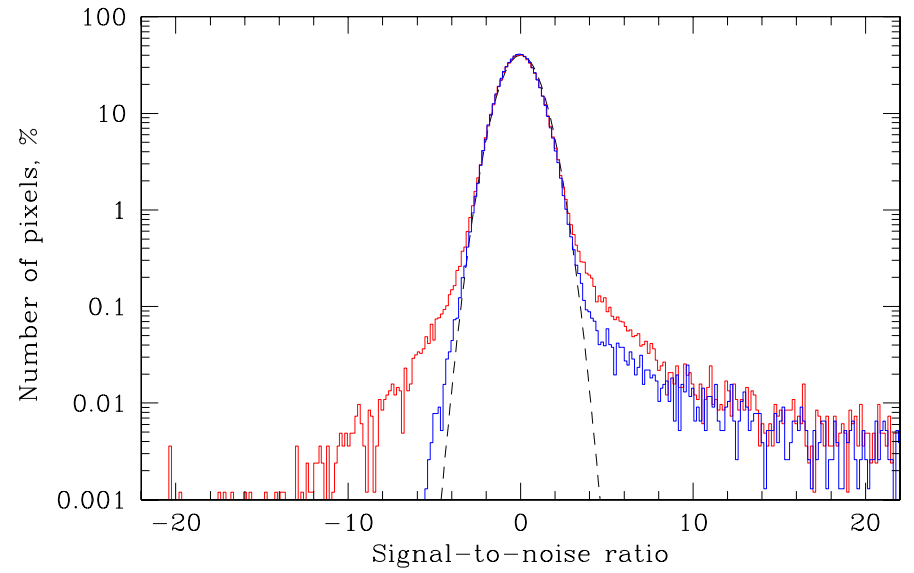

Fig. 4. Signal-to-noise ratio distribution of a number of pixels in the $17-60 \mathrm{keV}$ map of Fig. 3. Red and blue histograms show distributions of pixels in full and masked images, respectively. The dashed line represents the normal distribution with unit variance and zero mean.

image does not contain strong deviations from the Gaussian distribution, in contrast to the original one. Masked areas around bright sources reduce the geometrical area of the survey by $13 \%$ (Fig. 2).

Systematic effects are less important in the harder energy bands. Figure 5 shows that a sky image of the GC region in the $35-80 \mathrm{keV}$ energy range is practically free of the systematic noise with respect to $17-60 \mathrm{keV}$ band, which is confirmed by the $\mathrm{S} / \mathrm{N}$ distribution of image pixels in Fig. 6.

\subsection{Detection threshold}

Given the IBIS/ISGRI angular resolution of the survey, the sky map contains $\sim 3 \times 10^{5}$ statistically independent pixels. Taking into account the minor contribution of the systematic noise, we adopted a conservative detection threshold of $(S / N)_{\text {lim }}>4.7 \sigma$ to ensure that the final catalog contains no more than one spurious source assuming Poisson statistics.

A source detection in the region of $\sim 17$ degrees around GC should be interpreted with special care because of the possible false peaks induced by the systematic noise. The latter is revealed by a distorted shape that differs significantly from the instrumental point-spread function (PSF), which is a twodimensional Gaussian $\left(\sigma=5^{\prime}\right)$. The sky map of the GC region contains 12 peaks above $4.7 \sigma$ in the reference $17-60 \mathrm{keV}$ energy band. All these candidate sources have a very distorted shape, and none of them have been detected in the 35-80 keV energy range, which also points to a false detection. Therefore, all excesses in the GC region have been attributed to the systematic noise.

\section{Catalog of sources}

The catalog was compiled from the source sample exceeding the detection threshold in the reference $17-60 \mathrm{keV}$ energy band. The list of sources is presented in Table 2, and its content is described below.

Column (1) "Id" - source sequence number in the catalog.

Column (2) "Name" - source name. Their common names are given for sources whose nature was known before their detection by INTEGRAL. Sources discovered by INTEGRAL or those whose nature was established thanks to INTEGRAL observations are named "IGR"

Columns (3, 4) "RA, Dec" - source equatorial (J2000) coordinates.

Columns (5-7) "Flux" - time-averaged source flux in the 17-60 keV, 17-35 keV, and 35-80 keV energy bands, respectively. 
Table 2. Census of Galactic and extragalactic sources at $|b|<17.5^{\circ}$.

\begin{tabular}{lccccccc}
\hline \hline Energy range, keV & AGN & LMXB & HMXB & CV & Other & NotID & Total \\
\hline $17-60$ & $104+8^{s}$ & $100+8^{s}$ & $76+6^{s}$ & $35+1^{s}$ & 30 & 34 & 402 \\
$17-35$ & $89+7^{s}$ & $98+7^{s}$ & $74+6^{s}$ & $35+1^{s}$ & 28 & 22 & 367 \\
$35-80$ & $76+4^{s}$ & $80+5^{s}$ & $66+3^{s}$ & 13 & 26 & 7 & 280 \\
\hline
\end{tabular}

Notes. The number of sources with a tentative classification is denoted with $S$ index. Figure 7 shows the chart for source classes detected in the $17-60 \mathrm{keV}$ energy band.

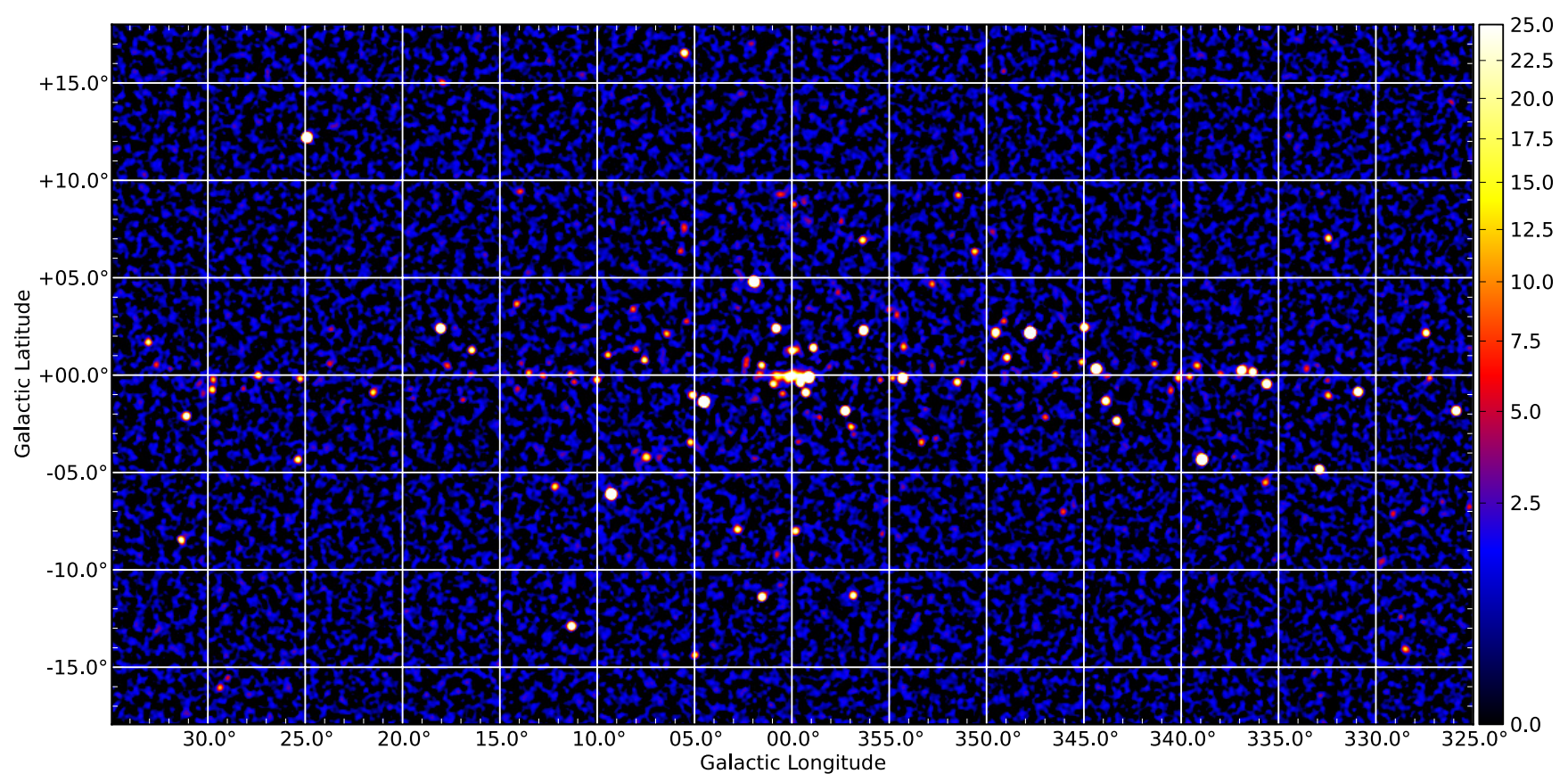

Fig. 5. INTEGRAL/IBIS hard X-ray (35-80 keV) map of the sky region around the GC. The total exposure is about $26 \mathrm{Ms}$ in the GC region. The image is shown in terms of $\mathrm{S} / \mathrm{N}$ with the color map ranging from values of 0 to 25 . This color scheme is used to emphasize sky background variations. Figure 6 demonstrates a corresponding S/N distribution of pixels.

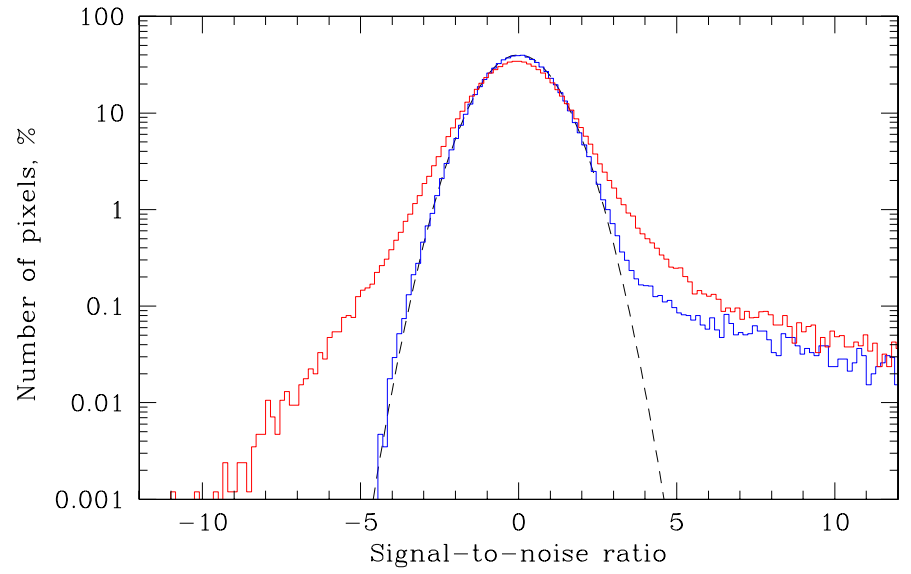

Fig. 6. Signal-to-noise ratio distribution of a number of pixels in the hard X-ray image shown in Fig. 5. The dashed line represents the normal distribution with unit variance and zero mean. Red and blue histograms show pixel distributions for images in the energy band $17-60 \mathrm{keV}$ (high systematic noise, see text) and 35-80 keV, respectively.

Column (8) "Type" - general astrophysical type of the object: LMXB (HMXB) - low- (high-) mass X-ray binary, AGN - active galactic nucleus, SNR - supernova remnant, $\mathrm{CV}$ - cataclysmic variable, PSR - isolated pulsar or pulsar wind nebula (PWN), SGR - soft gamma repeater, RS CVn coronally active binary star, SymbStar - symbiotic star, Cluster - cluster of galaxies. A question mark indicates that the specified type is not firmly determined, so should be confirmed.

Determining of the natures of the sources is complicated and a continually ongoing process. During the compilation of the catalog, we selected from our point of view, the most recent or reliable, identifications from the literature. In some cases (when references are not given), the identifications had been performed using recent observations of the Chandra, XMM-Newton, and Swift observatories, both the Simbad ${ }^{3}$ and NED ${ }^{4}$ database, as well as the 2 MASS catalog.

Column (9) "Ref." - references. These are mainly provided for new sources and are related to their discovery and/or nature. The papers describing a routine analysis of a given source e.g. confirmation of their nature, a refined position, etc. are not referenced.

Column (10) "Notes" - additional notes such as type subclass, redshift information, alternative source names. The redshifts of the extragalactic sources were obtained from the Simbad and NED databases.

\footnotetext{
${ }^{3}$ Simbad Astronomical Database http://simbad.u-strasbg.fr ${ }^{4}$ NASA/IPAC Extragalactic Database http://ned.ipac.caltech. edu
} 


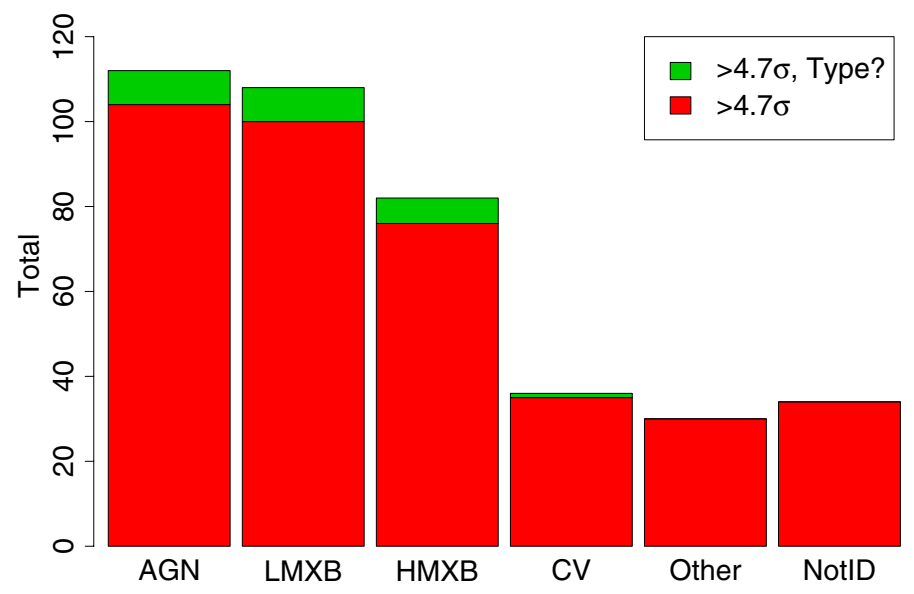

Fig. 7. Chart for the source classes detected at $S / N>4.7 \sigma$ in the reference $17-60 \mathrm{keV}$ energy range (see Table 2). Green bar denotes the number of sources that have a tentative association with a given type of objects.

\section{Concluding remarks}

Our Galactic survey is based on sky maps averaged over a nineyear time period, which obviously means that it has a strong bias against finding low $\mathrm{S} / \mathrm{N}$ transient sources. This ensures that the current survey contains mainly persistent objects, in addition to, however, objects with strong intrinsic variability.

We have presented detailed source statistics in Table 2 and a chart of source types in Fig. 7. Our Galactic survey has an identification completeness of $\left(N_{\text {Tot }}-N_{\text {NotID }}\right) / N_{\text {Tot }}=1-$ $34 / 402 \approx 0.92$, which provides a strong statistical basis for population studies. In the complementary paper of Lutovinov et al. (in prep.), we note in particular that we used the source sample and sensitivity maps of the current survey to study the HMXB luminosity function and their spatial distribution in the Galactic disk.

Acknowledgements. The data were obtained from the European and Russian INTEGRAL Science Data Centers ${ }^{5,6}$. This work was supported by the President of the Russian Federation (through the program supporting leading scientific schools, project NSH-5603.2012.2), by the Presidium of the Russian Academy of Sciences/RAS (program P21 "Non-stationary Phenomena in the Universe"), grants 12-02-01265, 11-02-01328 and 11-02-12285-ofim-2011 from Russian Foundation for Basic Research, State contract 14.740.11.0611. This research has made use of the IGR Sources page ${ }^{7}$ maintained by J. Rodriguez \& A. Bodaghee.

\section{References}

Ajello, M., Alexander, D. M., Greiner, J., et al. 2012, ApJ, in press [arXiv: 1202.3137$]$

Barlow, E. J., Knigge, C., Bird, A. J., et al. 2006, MNRAS, 372, 224

Barthelmy, S. D., Barbier, L. M., Cummings, J. R., et al. 2005, Space Sci. Rev., 120,143

Bassa, C. G., Jonker, P. G., in't Zand, J. J. M., \& Verbunt, F. 2006, A\&A, 446, $\mathrm{L} 17$

Bassani, L., Malizia, A., Stephen, J. B., et al. 2004, The Astronomer's Telegram, 232,1

Bassani, L., De Rosa, A., Bazzano, A., et al. 2005, ApJ, 634, L21

Bassani, L., Molina, M., Malizia, A., et al. 2006, ApJ, 636, L65

Bassani, L., Landi, R., Campana, R., et al. 2009, MNRAS, 395, L1

\footnotetext{
5 http://isdc.unige.ch

6 http://hea.iki.rssi.ru/rsdc

7 http://irfu.cea.fr/Sap/IGR-Sources/
}

Baumgartner, W. H., Tueller, J., Markwardt, C., \& Skinner, G. 2010, BAAS, 42, 675

Beckmann, V., Kennea, J. A., Markwardt, C., et al. 2005, ApJ, 631, 506

Beckmann, V., Soldi, S., Ricci, C., et al. 2009, A\&A, 505, 417

Bikmaev, I. F., Sunyaev, R. A., Revnivtsev, M. G., \& Burenin, R. A. 2006a, Astron. Lett., 32, 221

Bikmaev, I. F., Revnivtsev, M. G., Burenin, R. A., \& Sunyaev, R. A. 2006b, Astron. Lett., 32, 588

Bikmaev, I. F., Burenin, R. A., Revnivtsev, M. G., et al. 2008, Astron. Lett., 34, 653

Bird, A. J., Barlow, E. J., Bassani, L., et al. 2006, ApJ, 636, 765

Bird, A. J., Malizia, A., Bazzano, A., et al. 2007, ApJS, 170, 175

Bird, A. J., Bazzano, A., Bassani, L., et al. 2010, ApJS, 186, 1

Bodaghee, A., Walter, R., Zurita Heras, J. A., et al. 2006, A\&A, 447, 1027

Bodaghee, A., Courvoisier, T. J.-L., Rodriguez, J., et al. 2007, A\&A, 467, 585

Bodaghee, A., Tomsick, J. A., Rodriguez, J., \& James, J. B. 2012, ApJ, 744, 108

Brandt, S., Budtz-Jørgensen, C., \& Chenevez, J. 2006a, The Astronomer's Telegram, 778, 1

Brandt, S., Budtz-Jorgensen, C., Chenevez, J., et al. 2006b, The Astronomer's Telegram, 970, 1

Brandt, S., Budtz-Jørgensen, C., Gotz, D., Hurley, K., \& Frontera, F. 2007, The Astronomer's Telegram, 1054, 1

Burenin, R., Mescheryakov, A., Revnivtsev, M., Bikmaev, I., \& Sunyaev, R. 2006, The Astronomer's Telegram, 880, 1

Burenin, R., Revnivtsev, M., Mescheryakov, A., et al. 2007, The Astronomer's Telegram, 1270, 1

Burenin, R. A., Mescheryakov, A. V., Revnivtsev, M. G., et al. 2008, Astron. Lett., 34, 367

Butler, S. C., Tomsick, J. A., Chaty, S., et al. 2009, ApJ, 698, 502

Bélanger, G., Goldwurm, A., Renaud, M., et al. 2006, ApJ, 636, 275

Chaty, S., Rahoui, F., Foellmi, C., et al. 2008, A\&A, 484, 783

Chelovekov, I. V., \& Grebenev, S. A. 2007, Astron. Lett., 33, 807

Chernyakova, M., Lutovinov, A., Capitanio, F., Lund, N., \& Gehrels, N. 2003, The Astronomer's Telegram, 157, 1

Chernyakova, M., Courvoisier, T. J.-L., Rodriguez, J., \& Lutovinov, A. 2005a, The Astronomer's Telegram, 519, 1

Chernyakova, M., Lutovinov, A., Rodríguez, J., \& Revnivtsev, M. 2005b, MNRAS, 364, 455

Churazov, E., Sunyaev, R., Revnivtsev, M., et al. 2007, A\&A, 467, 529

Goncalves, T. S., Martin, D. C., Halpern, J. P., Eracleous, M., \& Pavlov, G. G. 2008, The Astronomer's Telegram, 1623, 1

Courvoisier, T. J.-L., Walter, R., Rodriguez, J., Bouchet, L., \& Lutovinov, A. A. 2003, IAU Circ., 8063, 3

Cusumano, G., La Parola, V., Segreto, A., et al. 2010, A\&A, 510, A48

Curran, P. A., Chaty, S., \& Zurita Heras, J. A. 2011a, A\&A, 533, A3

Curran, P. A., Chaty, S., Zurita Heras, J. A., Tomsick, J. A., \& Maccarone, T. J. 2011b, MNRAS, 417, L26

Degenaar, N., Jonker, P. G., Torres, M. A. P., et al. 2010, MNRAS, 404, 1591

Degenaar, N., Altamirano, D., Wijnands, R. 2012, The Astronomer's Telegram, 4219, 1

Den Hartog, P. R., Kuiper, L., Hermsen, W., et al. 2005, The Astronomer's Telegram, 394, 1

Del Monte, E., Evangelista, Y., Feroci, M., et al. 2008, The Astronomer's Telegram, 1445, 1

Donato, D., Sambruna, R. M., \& Gliozzi, M. 2005, A\&A, 433, 1163

Eckert, D., Walter, R., Kretschmar, P., et al. 2004, The Astronomer's Telegram, 352,1

Fiocchi, M., Bassani, L., Bazzano, A., et al. 2010, ApJ, 720, 987

Funk, S., Hinton, J. A., Moriguchi, Y., et al. 2007, A\&A, 470, 249

Gehrels, N., Chincarini, G., Giommi, P., et al. 2004, ApJ, 611, 1005

Gibaud, L., Bazzano, A., Bozzo, E., et al. 2011, The Astronomer's Telegram, 3565,1

Grebenev, S. A., \& Sunyaev, R. A. 2004, The Astronomer's Telegram, 342, 1

Grebenev, S. A., \& Sunyaev, R. A. 2007, Astron. Lett., 33, 149

Grebenev, S. A., Ubertini, P., Chenevez, J., Orr, A., \& Sunyaev, R. A. 2004, The Astronomer's Telegram, 275, 1

Grebenev, S. A., Molkov, S. V., \& Sunyaev, R. A. 2005a, The Astronomer's Telegram, 444, 1

Grebenev, S. A., Bird, A. J., Molkov, S. V., et al. 2005b, The Astronomer's Telegram, 457, 1

Grebenev, S. A., Molkov, S. V., \& Sunyaev, R. A. 2005c, The Astronomer's Telegram, 467, 1

Grebenev, S. A., Molkov, S. V., Revnivtsev, M. G., \& Sunyaev, R. A. 2007, ESA Spec. Publ., 622, 373

Gros, A., Goldwurm, A., Cadolle-Bel, M., et al. 2003, A\&A, 411, L179

Gänsicke, B. T., Marsh, T. R., Edge, A., et al. 2005, MNRAS, 361, 141

Harrison, F. A., Boggs, S., Christensen, F., et al. 2010, Proc. SPIE, 7732 
den Hartog, P. R., Hermsen, W., Kuiper, L. M., et al. 2004a, The Astronomer's Telegram, 261, 1

den Hartog, P. R., Kuiper, L. M., Corbet, R. H. D., et al. 2004b, The Astronomer's Telegram, 281, 1

Halpern, J. P. 2006, The Astronomer's Telegram, 847, 1

Hannikainen, D. C., Rodriguez, J., \& Pottschmidt, K. 2003, IAU Circ., 8088, 4

Heinke, C. O., Tomsick, J. A., Yusef-Zadeh, F., \& Grindlay, J. E. 2009, ApJ, 701, 1627

Hiemstra, B., Méndez, M., Done, C., et al. 2011, MNRAS, 411, 137

Kalamkar, M., Homan, J., Altamirano, D., et al. 2011, ApJ, 731, L2

Karasev, D., Tsygankov, S., Lutovinov, A., Churazov, E., \& Sunyaev, R. 2007a, The Astronomer's Telegram, 1245, 1

Karasev, D. I., Lutovinov, A. A., \& Grebenev, S. A. 2007b, Astron. Lett., 33, 159

Karasev, D. I., Lutovinov, A. A., \& Burenin, R. A. 2010, MNRAS, 409, L69

Karasev, D. I., Lutovinov, A. A., Revnivtsev, M. G., \& Krivonos, R. A. 2012, Astron, Letters, 38, N10, in press

Keek, S., Kuiper, L., \& Hermsen, W. 2006, The Astronomer's Telegram, 810, 1

Kretschmar, P., Mereghetti, S., Hermsen, W., et al. 2004, The Astronomer's Telegram, 345,1

Krivonos, R., Vikhlinin, A., Churazov, E., et al. 2005, ApJ, 625, 89

Krivonos, R., Revnivtsev, M., Churazov, E., et al. 2007a, A\&A, 463, 957

Krivonos, R., Revnivtsev, M., Lutovinov, A., et al. 2007b, A\&A, 475, 775

Krivonos, R., Revnivtsev, M., Tsygankov, S., et al. 2010a, A\&A, 519, A107

Krivonos, R., Tsygankov, S., Revnivtsev, M., et al. 2010b, A\&A, 523, A61

Krivonos, R., Tsygankov, S., Burenin, R., Revnivtsev, M., \& Lutovinov, A. 2011, The Astronomer's Telegram, 3382, 1

Kuiper, L., Hermsen, W., in't Zand, J., \& den Hartog, P. R. 2005, The Astronomer's Telegram, 662, 1

Kuiper, L., Keek, S., Hermsen, W., Jonker, P. G., \& Steeghs, D. 2006a, The Astronomer's Telegram, 684, 1

Kuiper, L., den Hartog, P. R., \& Hermsen, W. 2006b, The Astronomer's Telegram, 939, 1

Kuiper, L., Jonker, P. G., Torres, M. A. P., Rest, A., \& Keek, S. 2008, The Astronomer's Telegram, 1774, 1

Kuulkers, E., Lutovinov, A., Parmar, A., et al. 2003, The Astronomer's Telegram, 149,1

Kuulkers, E., Shaw, S., Paizis, A., et al. 2006, The Astronomer's Telegram, 874, 1

Landi, R., Bassani, L., Malizia, A., et al. 2010, MNRAS, 403, 945

Landi, R., Bassani, L., Masetti, N., Bazzano, A., \& Bird, A. J. 2011a, The Astronomer's Telegram, 3271, 1

Landi, R., Bassani, L., Malizia, J. B., et al. 2011b, Published in Proceedings of the Workshop The Extreme and Variable High Energy Sky, Chia Laguna, Cagliari, Italy, September 19-23, PoS, 009

Landi, R., Bassani, L., Masetti, N., et al. 2012, The Astronomer's Telegram, 4233,1

Leyder, J.-C., Walter, R., \& Rauw, G. 2008, A\&A, 477, L29

Linares, M., Watts, A. L., Wijnands, R., et al. 2009, MNRAS, 392, L11

Lutovinov, A. A., \& Revnivtsev, M. G. 2003a, Astron. Lett., 29, 719

Lutovinov, A., Shaw, S., Foschini, L., \& Paul, J. 2003b, The Astronomer's Telegram, 154,1

Lutovinov, A., Walter, R., Belanger, G., et al. 2003c, The Astronomer's Telegram, 155, 1

Lutovinov, A., Cadolle Bel, M., Belanger, G., et al. 2004a, The Astronomer's Telegram, 328, 1

Lutovinov, A., Rodrigues, J., Budtz-Jorgensen, C., Grebenev, S., \& Winkler, C. 2004b, The Astronomer's Telegram, 329, 1

Lutovinov, A., Rodriguez, J., Revnivtsev, M., \& Shtykovskiy, P. 2005a, A\&A 433, L41

Lutovinov, A., Revnivtsev, M., Gilfanov, M., et al. 2005b, A\&A, 444, 821

Lutovinov, A., Revnivtsev, M., Gilfanov, M., \& Sunyaev, R. 2007, ESA Spec. Publ., 622, 241

Lutovinov, A., Burenin, R., Sazonov, S., et al. 2010a, The Astronomer's Telegram, 2759, 1

Lutovinov, A. A., Burenin, R. A., Revnivtsev, M. G., Suleimanov, V. F., \& Tkachenko, A. Y. 2010b, Astron. Lett., 36, 904

Lutovinov, A. A., Burenin, R. A., Revnivtsev, M. G., \& Bikmaev, I. F. 2012, Astron. Lett., 38,1

Malizia, A., Bassani, L., Stephen, J. B., et al. 2005, ApJ, 630, L157

Malizia, A., Bassani, L., Sguera, V., et al. 2010, MNRAS, 408, 975

Martí, J., Paredes, J. M., Bloom, J. S., et al. 2004, A\&A, 413, 309

Markwardt, C. B. 2008, The Astronomer's Telegram, 1686, 1

Markwardt, C. B., Swank, J. H., \& Strohmayer, T. E. 2004, The Astronomer's Telegram, 353, 1

Masetti, N., Palazzi, E., Bassani, L., Malizia, A., \& Stephen, J. B. 2004, A\&A, 426, L41

Masetti, N., Bassani, L., Bird, A. J., \& Bazzano, A. 2005, The Astronomer's Telegram, 528, 1
Masetti, N., Pretorius, M. L., Palazzi, E., et al. 2006a, A\&A, 449, 1139

Masetti, N., Bassani, L., Bazzano, A., et al. 2006b, A\&A, 455, 11

Masetti, N., Morelli, L., Palazzi, E., et al. 2006c, A\&A, 459, 21

Masetti, N., Bassani, L., Dean, A. J., Ubertini, P., \& Walter, R. 2006d, The Astronomer's Telegram, 715, 1

Masetti, N., Morelli, L., Palazzi, E., et al. 2006e, The Astronomer's Telegram, 783,1

Masetti, N., Landi, R., Pretorius, M. L., et al. 2007a, A\&A, 470, 331

Masetti, N., Cellone, S. A., Landi, R., et al. 2007b, The Astronomer's Telegram, 1034,1

Masetti, N., Rigon, E., Maiorano, E., et al. 2007c, A\&A, 464, 277

Masetti, N., Mason, E., Morelli, L., et al. 2008, A\&A, 482, 113

Masetti, N., Parisi, P., Palazzi, E., et al. 2009, A\&A, 495, 121

Masetti, N., Landi, R., Sguera, V., et al. 2010a, A\&A, 511, A48

Masetti, N., Parisi, P., Palazzi, E., et al. 2010b, A\&A, 519, A96

Masetti, N., Parisi, P., Jiménez-Bailón, E., et al. 2012a, A\&A, 538, A123

Masetti, N., Landi, R., Parisi, P., et al. 2012b, The Astronomer's Telegram, 4248,

McGlynn, T., Scollick, K., \& White, N. 1998, New Horizons from Multi-Wavelength Sky Surveys, 179, 465

Mescheryakov, A., Burenin, R., Sazonov, S., et al. 2009, The Astronomer's Telegram, 2132, 1

Milisavljevic, D., Fesen, R. A., Parrent, J. T., \& Thorstensen, J. R. 2011, The Astronomer's Telegram, 3146, 1

Molkov, S., Mowlavi, N., Goldwurm, A., et al. 2003, The Astronomer's Telegram, 176, 1

Molkov, S. V., Cherepashchuk, A. M., Lutovinov, A. A., et al. 2004, Astron. Lett., 30,534

Morelli, L., Masetti, N., Bassani, L., et al. 2006, The Astronomer's Telegram, 785,1

Nespoli, E., Fabregat, J., \& Mennickent, R. E. 2008a, The Astronomer's Telegram, 1396, 1

Nespoli, E., Fabregat, J., \& Mennickent, R. E. 2008b, A\&A, 486, 911

Nespoli, E., Fabregat, J., \& Mennickent, R. E. 2010, A\&A, 516, A94

Nucita, A. A., Carpano, S., \& Guainazzi, M. 2007, A\&A, 474, L1

Nucita, A. A., De Paolis, F., Saxton, R., \& Read, A. M. 2012, New A, 17, 589

Parisi, P., Masetti, N., Bassani, L., et al. 2012, The Astronomer's Telegram, 4151, 1

Paizis, A., Nowak, M. A., Chaty, S., et al. 2007a, ApJ, 657, L109

Paizis, A., Beckmann, V., Gotz, D., et al. 2007b, The Astronomer's Telegram, 1248,1

Produit, N., Ballet, J., \& Mowlavi, N. 2004, The Astronomer's Telegram, 278, 1

Ratti, E. M., Bassa, C. G., Torres, M. A. P., et al. 2010, MNRAS, 408, 1866

Reig, P., \& Roche, P. 1999, MNRAS, 306, 100

Renaud, M., Marandon, V., Gotthelf, E. V., et al. 2010b, ApJ, 716, 663

Revnivtsev, M., Chernyakova, M., Capitanio, F., et al. 2003a, The Astronomer's Telegram, 132, 1

Revnivtsev, M. G., Sazonov, S. Y., Gilfanov, M. R., \& Sunyaev, R. A. 2003b, Astron. Lett., 29, 587

Revnivtsev, M., Tuerler, M., Del Santo, M., et al. 2003c, IAU Circ., 8097, 2

Revnivtsev, M. G., Sazonov, S. Y., Gilfanov M. R., \& Sunyaev, R. A. 2003d, Astron. Lett., 29, 587

Revnivtsev, M. G., Churazov, E. M., Sazonov, S. Yu., et al. 2004a, A\&A, 425, L49

Revnivtsev, M. G., Sunyaev, R. A., Varshalovich, D. A., et al. 2004b, Astron Lett., 30,382

Revnivtsev, M. G., Sazonov, S. Y., Molkov, S. V., et al. 2006, Astron. Lett., 32 , 145

Revnivtsev, M., Lutovinov, A., Churazov, E., et al. 2008a, A\&A, 491, 209

Revnivtsev, M., Sazonov, S., Krivonos, R., Ritter, H., \& Sunyaev, R. 2008b, A\&A, 489, 1121

Revnivtsev, M. G., Kniazev, A. Yu., Sazonov, S. Yu., et al. 2009, Astron. Lett., 35,33

Reynolds, M. T., Miller, J. M., Maitra, D., et al. 2012, The Astronomer's Telegram, 3951, 1

Rodriguez, J., Garau, A. D., Grebenev, S., et al. 2004, The Astronomer's Telegram, 340, 1

Sazonov, S., Churazov, E., Revnivtsev, M., Vikhlinin, A., \& Sunyaev, R. 2005, A\&A, 444, L37

Sazonov, S., Revnivtsev, M., Krivonos, R., Churazov, E., \& Sunyaev, R. 2007, A\&A, 462,57

Sazonov, S., Revnivtsev, M., Burenin, R., et al. 2008, A\&A, 487, 509

Scaringi S., Bird, A. J., Norton, A. J., et al. 2010, MNRAS, 401, 2207

Sguera, V., Bazzano, A., Bird, A. J., et al. 2006, ApJ, 646, 452

Smith, N., \& Hartigan, P. 2006, ApJ, 638, 1045

Soldi, S., Brandt, S., Garau, A. D., et al. 2005, The Astronomer's Telegram, 456,1

Soldi, S., Walter, R., Eckert, D., et al. 2006, The Astronomer's Telegram, 885, 1 
R. Krivonos et al.: INTEGRAL 9-year Galactic survey

Steeghs, D., Knigge, C., Drew, J., Unruh, Y., \& Greimel, R. 2008, The Astronomer's Telegram, 1653, 1

Sunyaev, R., Lutovinov, A., Molkov, S., \& Deluit, S. 2003a, The Astronomer's Telegram, 181, 1

Sunyaev, R. A., Grebenev, S. A., Lutovinov, A. A., et al. 2003b, The Astronomer's Telegram, 190, 1

Takahashi, T., Mitsuda, K., Kelley, R., et al. 2010, Proc. SPIE, 7732

Terrier, R., Mattana, F., Djannati-Atai, A., et al. 2008, AIP Conf. Ser., 1085, 312

Tomsick, J. A., Lingenfelter, R., Walter, R., et al. 2003, IAU Circ., 8076, 1

Tomsick, J. A., Lingenfelter, R., Corbel, S., Goldwurm, A., \& Kaaret, P. 2004, The Astronomer's Telegram, 224, 1

Tomsick, J. A., Chaty, S., Rodriguez, J., et al. 2006, ApJ, 647, 1309

Tomsick, J. A., Chaty, S., Rodriguez, J., Walter, R., \& Kaaret, P. 2008, ApJ, 685, 1143

Tomsick, J. A., Chaty, S., Rodriguez, J., Walter, R., \& Kaaret, P. 2009, ApJ, 701, 811

Torrejón, J. M., Negueruela, I., Smith, D. M., \& Harrison, T. E. 2010, A\&A, 510, A61

Torres, M. A. P., Garcia, M. R., McClintock, J. E., et al. 2004, The Astronomer's Telegram, 264, 1
Torres, M. A. P., Steeghs, D., Garcia, M. R., et al. 2006, The Astronomer's Telegram, 862, 1

Torres, M. A. P., Steeghs, D., Jonker, P. G., et al. 2007, The Astronomer's Telegram, 1286, 1

Tueller, J., Baumgartner, W. H., Markwardt, C. B., et al. 2010, ApJS, 186, 378

Tuerler, M., Walter, R., \& Ferrigno, C. 2012, The Astronomer's Telegram, 4183, 1

Ubertini P., Lebrun, F., Di Cocco, G., et al. 2003, A\&A, 411, L131

Ubertini, P., Bassani, L., Malizia, A., et al. 2005, ApJ, 629, L109

Walter, R., Bodaghee, A., Barlow, E. J., et al. 2004, The Astronomer's Telegram, 229, 1

Walter, R., Zurita Heras, J., Bassani, L., et al. 2006, A\&A, 453, 133

Watson, M. G., Schröder, A. C., Fyfe, D., et al. 2009, A\&A, 493, 339

Wijnands, R. 2006, The Astronomer's Telegram, 972, 1

Winkler, C., Courvoisier, T. J.-L., Di Cocco, G., et al. 2003, A\&A, 411, L1

Winter, L. M., Mushotzky, R. F., Tueller, J., \& Markwardt, C. 2008, ApJ, 674, 686

Zurita Heras, J. A., Chaty, S., \& Tomsick, J. A. 2009, A\&A, 502, 787

in't Zand, J. J. M. 2005, A\&A, 441, L1

Zolotukhin, I. Y., \& Revnivtsev, M. G. 2011, MNRAS, 411, 620 